\title{
Accelerated Testing of Advanced Battery Technologies in PHEV Applications
}

\author{
Loïc Gaillac*
}

EPRI and DaimlerChrysler developed a Plug-in Hybrid Electric Vehicle (PHEV) using the Sprinter Van to reduce emissions, fuel consumption, and operating costs while maintaining equivalent or superior functionality and performance. The utilization of grid electricity and operating efficiency significantly reduce petroleum consumption, greenhouse gas emissions, and criteria pollutants, especially benefiting urban areas.

Southern California Edison (SCE) collaborated with EPRI and DaimlerChrysler to evaluate the vehicle and two different battery technologies. This paper documents one of the first published battery test results related to PHEV applications from the beginning of the project, in September 2004.

The partners selected two advanced battery technologies, lithium ion (Li-Ion) chemistry from SAFT and nickel metal hydride (NiMH) chemistry from VARTA. The primary goals of the test are to evaluate the performance and cycle life of traction batteries for future PHEV Sprinter Van production using PHEV test profiles. The test profile replicates the most demanding urban driving conditions for the battery (i.e., low speed, high acceleration in charge-sustained HEV mode at low battery state of charge). It also uses a combination of HEV and EV driving modes to represent over $50 \%$ of statistical daily trips. One cycle includes three modes: (1) charge depletion mode, simulating the EV operation, (2) charge sustained mode, simulating the HEV operation, and (3) recharge mode, simulating the plug-in operation.

The SAFT battery technology shows encouraging results from simulated PHEV tests; one test cycle represents an average daily operation of a PHEV Sprinter Van (i.e., a 2.6-hour, 50-mile drive).

Keywords: Li-Ion, SAFT, PHEV, Battery, Life-Cycle

\section{INTRODUCTION}

The DaimlerChrysler Sprinter Van is a multipurpose commercial van designed for a number of diverse tasks. The vehicle is available in numerous configurations including panel van, passenger van, ambulance, work truck, and shuttle bus. The Sprinter is available in wheelbase lengths of 3.0 to 4.0 meters with gross vehicle weight (GVW) of up to 6.0 metric tons.

EPRI and DaimlerChrysler developed a Plug-in Hybrid Electric Vehicle (PHEV) concept for the Sprinter in an effort to reduce the emissions, fuel consumption, and operating costs of the vehicle while maintaining equivalent or superior functionality and performance. The PHEV Sprinter was designed to achieve 20 miles $(32 \mathrm{~km})$ of operating range in its pure electric mode. The vehicle design also featured a charge-sustaining hybrid operating mode with up to $50 \%$ higher fuel

* Advanced Energy Storage Lead Engineer

Electric Transportation

Southern California Edison economy than the conventional Sprinter vehicle.

It is projected that the utilization of grid electricity combined with the improved efficiency will significantly reduce the greenhouse gas emissions and criteria pollutants produced by the vehicle, especially in dense urban areas. SCE partnered with EPRI and DaimlerChrysler to evaluate the vehicle and two different battery types. The effort was split into two projects, vehicle evaluation and battery testing. The primary objective is to demonstrate the capability of the PHEV Sprinter concept to provide environmental benefits while reducing lifecycle operating costs in a typical commercial setting.

This paper documents the work performed since the start of the project in September 2004 to May 2007. Based on the excellent data collected, the project was extended to December 2007.

\section{PROJECT DISCUSSION}

\subsection{Applications}


The primary goals of this project are to evaluate the performance and cycle life of traction batteries intended for future Sprinter vehicle production under PHEV test profiles.

\subsection{Battery Technology Selected}

\subsubsection{GENERAL DESCRIPTION}

Two advanced battery technologies have been selected for this project. The lithium ion (Li-Ion) chemistry from SAFT (Figure 1) and the nickel metal hydride (NiMH) chemistry from VARTA. Only the results from the $\mathrm{Li}^{-}$ Ion battery test will be discussed in this paper.

\subsubsection{SPECIFICATIONS}

The manufacturer's specification is listed in Table 1.

\section{TEST PLAN}

\subsection{Test Procedure Development}

\subsubsection{TEST PROFILE}

The test profile is derived from a dynamic vehicle-level simulation based on the final portion of the INRETS URB1 vehicle test cycle. The cycle replicates the urban driving conditions likely to be the most demanding to the battery (low speed, high acceleration and chargesustained HEV mode at low battery SOC). It also uses a combination of both $\mathrm{HEV}$ and $\mathrm{EV}$ driving modes to represent greater than $50 \%$ of statistical daily trips.

The EV and HEV profiles are shown in Figure 2 and Figure 3.

\subsubsection{TEST PROCEDURE IMPLEMENTATION}

The test protocol is composed of three modes. The charge depletion mode, simulating the EV operation, the charge sustained mode, simulating the HEV operation, and the recharge mode, simulating the plugin operation. One test cycle includes all three modes. The test profiles are scaled to match the number of battery modules / blocks to be tested.

All battery safety limits, as stated by the manufacturer, have to be respected during the test cycles.

\subsubsection{Charge Depletion Mode}

The charge depletion (CD) mode starts with a fully charged battery. The 181.5 second EV test profile is continuously applied to the battery until the battery voltage drops below a pre-defined threshold for 10

\begin{tabular}{|l|c|}
\hline Manufacturer & SAFT \\
\hline Battery Chemistry & Li-Ion \\
\hline Module / Block Level & \\
\hline Package & 6-cell module \\
\hline Module / Block Nominal Voltage (V) & 21.6 \\
\hline Cell Capacity (@C/1, Ah) & 39 \\
\hline Module / Block Energy (@ C/3, Wh) & 912 \\
\hline Dimension (mm) & $190 \times 123 \times 242$ \\
\hline Weight (kg) & 8 \\
\hline Vehicle Pack Level & \\
\hline No. of Module / Block & 17 \\
\hline No. of Cells & 102 \\
\hline Nominal Voltage (V) & 367 \\
\hline Pack Energy (@ C/3, kWh) & 15.5 \\
\hline Battery Cooling & Liquid Cooled \\
\hline
\end{tabular}

Table 1: Battery specifications

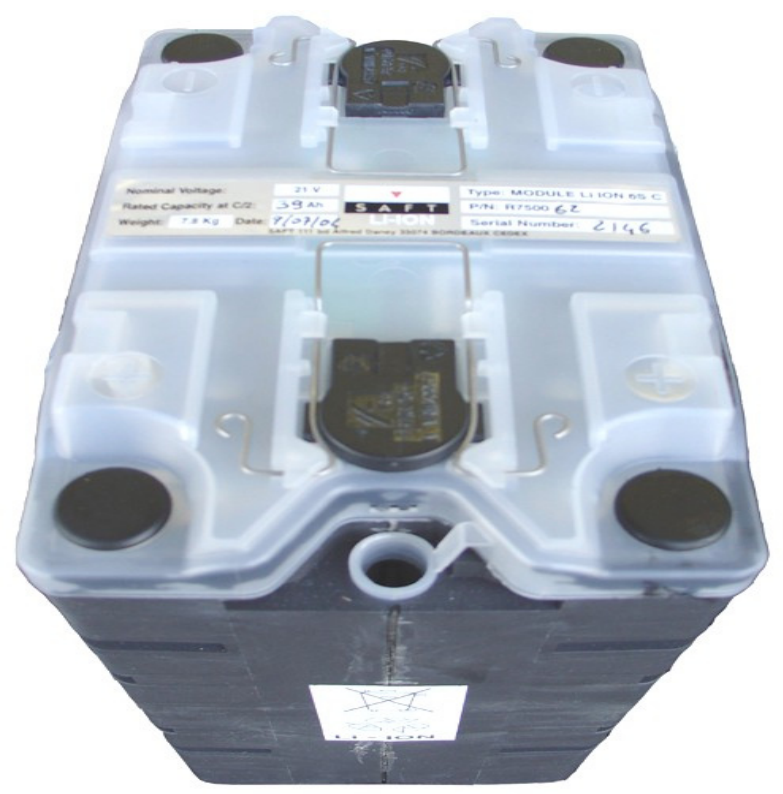

Figure 1: SAFT Li-Ion battery module

consecutive seconds. This threshold is determined at the beginning of the life cycle test to ensure that the battery SOC at the end of the charge depletion mode is approximately $25 \%$. 
3.1.2.2 Charge Sustained Mode

The charge sustained mode (CS) starts at the completion of the CD mode. The 181.5 second HEV test profile is continually applied to the battery until the total duration of the CD mode and the CS mode has reached a combined 2.6 hours.

The battery is then allowed to rest for 15 minutes before the next mode.

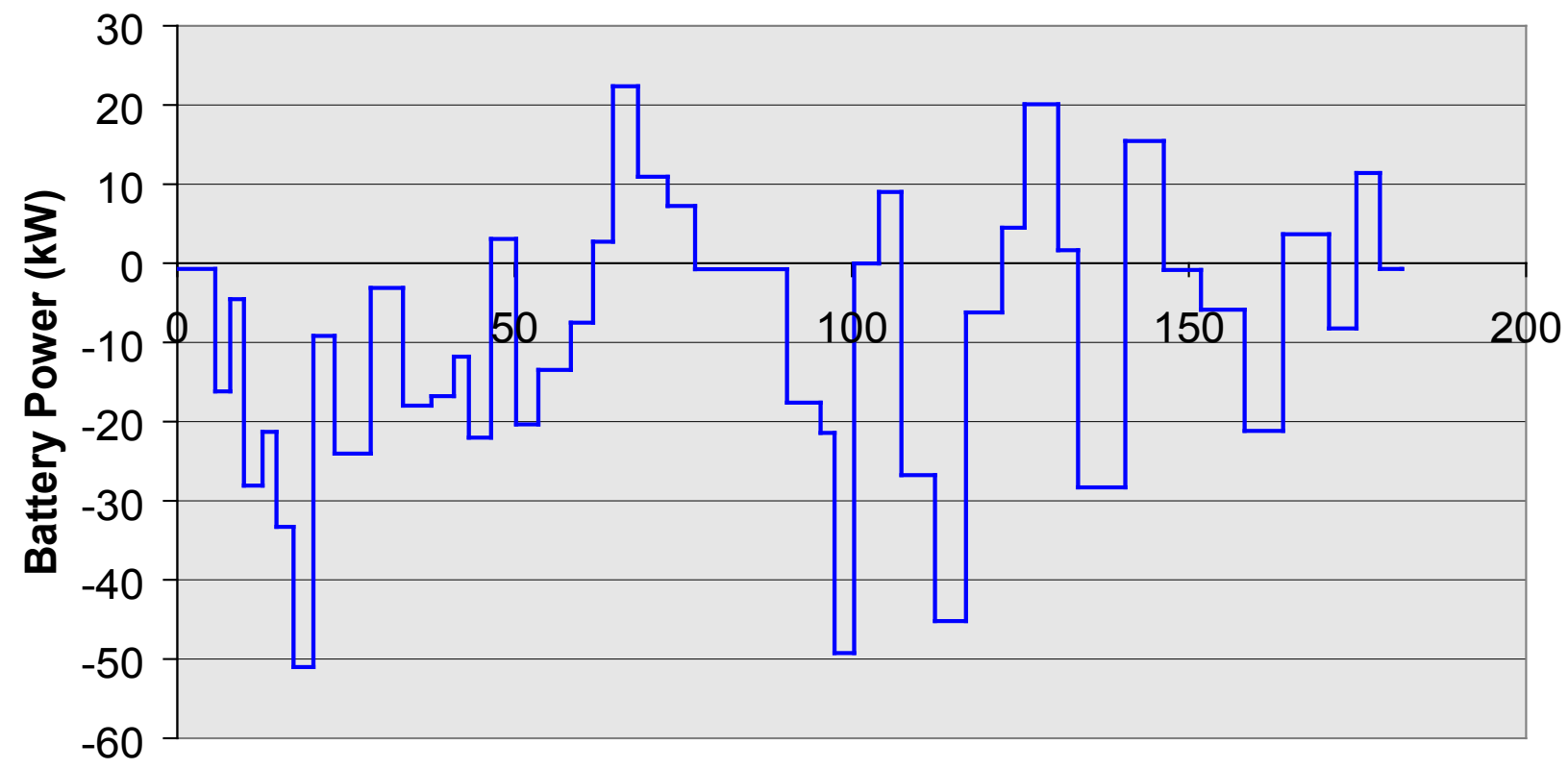

Time (s)

Figure 2: EV mode test profile

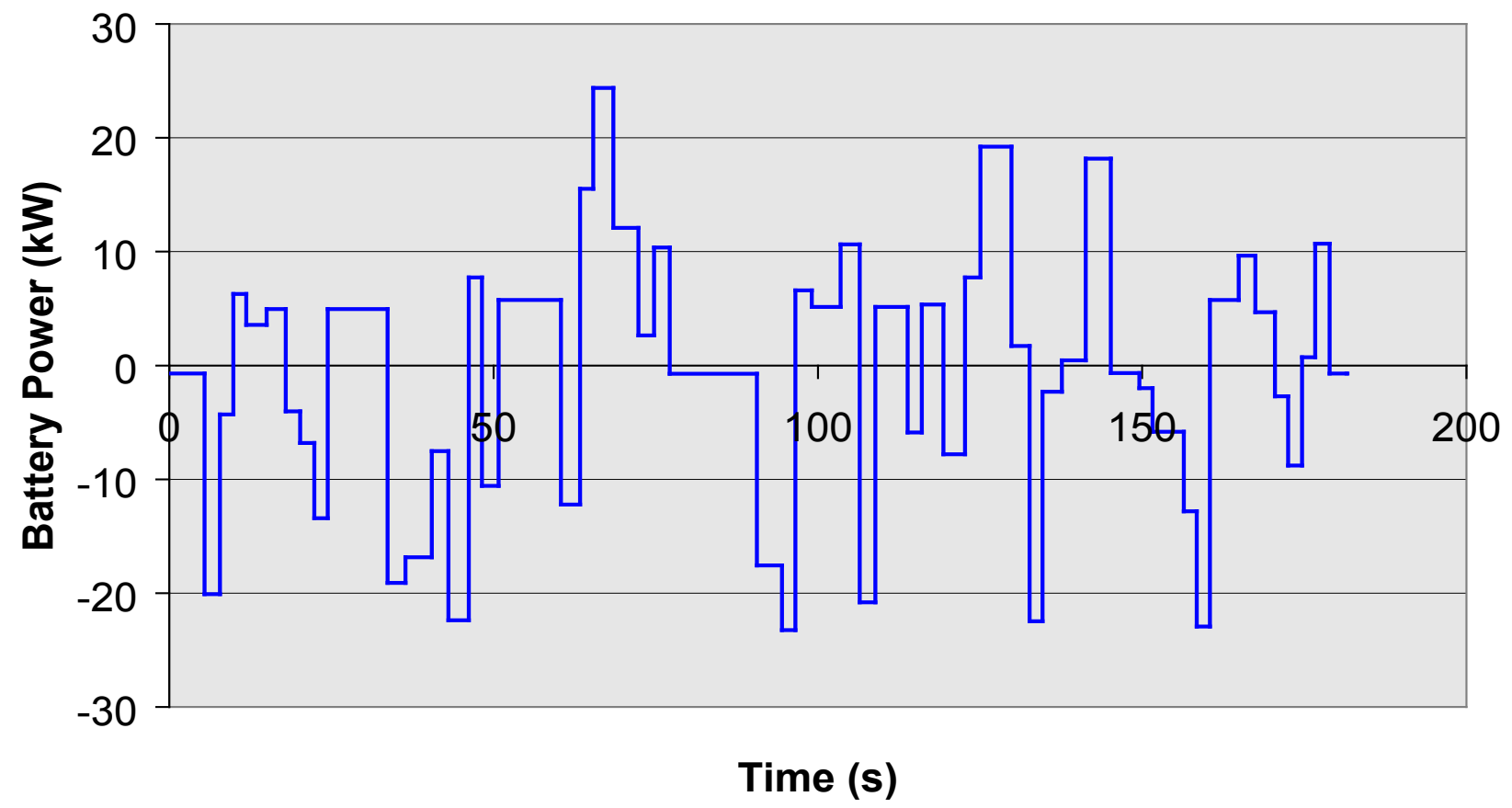

Figure 3: HEV mode test profile

A negative battery power value indicates that the battery is being discharged 
In the CS mode, the battery SOC should remain constant. In order to ensure that the net energy transfer between the battery and the test equipment remains equal to zero, the generic HEV test profile is adjusted. A minor offset is applied to the test profile at the beginning of the life cycle test to guarantee a constant SOC during the CS mode.

Throughout the course of the test, the SOC may drift as the battery internal resistance is changing. If the balance of capacity in ampere-hours exceeds a predefined parameter at the end of any test profile, the SOC will be readjusted automatically.

\subsubsection{Recharge Mode (or Charge Mode)}

In this mode, the battery is charged using the manufacturer's suggested charge algorithm; this is the highest rate that will not present any detrimental effect on the battery life. The charging duration is 3.5 hours. At the completion of this mode, a 30 minute rest period is allowed before the start of a new test cycle.

\subsubsection{REFERENCE PERFORMANCE TESTS}

Reference Performance Tests (RPTs) are conducted before the start of the life cycle test, and at periodic intervals to characterize the performance of the battery.

The following tests are included:

o A constant current discharge at a rate of $\mathrm{C}_{1} / 1$

o A constant current discharge at a rate of $\mathrm{C}_{1} / 3$

o A peak power test

o A HPPC (Hybrid Pulse Power Characterization) test (performed in the dual mode configuration)

The first three tests are based on the USABC Electric Vehicle Test Procedure Manual (Revision 2) [1]; the fourth test is based on the PNGV Battery Test Manual (Revision 3) [2]

A preliminary cycle, including a discharge at a constant current rate of $\mathrm{C}_{1} / 3$ down to $60 \%$ DOD and a full recharge, is performed prior to the RPT. A 30minute rest is included in between each charge and discharge.

\subsubsection{TEST CYCLE SUMMARY}

The average duration of one single test cycle is approximately six hours. Therefore, a total of approximately four test cycles can be applied every day. Each test cycle applied to the battery simulates the daily operation of the Sprinter PHEV in the field. The CS and CD modes are equivalent to a 50-mile/2.6- hour drive with the Sprinter PHEV.

Periodic RPTs are performed every 240 test cycles, equivalent to approximately two months of testing.

\subsection{Life Cycle Tests}

The batteries are tested using a computer-controlled automated battery cycler.

The tests are performed in temperature-controlled environmental test chambers. In addition to having safety temperature limit controllers, each chamber is equipped with smoke and heat detectors connected to the building fire alarm panel.

\subsubsection{SAFT LI-ION BATTERY}

\subsubsection{Test Setup}

The SAFT Li-Ion battery pack is composed of 17 modules of six cells. Three 6-cell modules are currently tested. A recirculation chiller is used to provide continuous flow of cooling fluid at a temperature of $25^{\circ} \mathrm{C}$ and a flow rate of $0.5 \mathrm{l} / \mathrm{min}$. The battery pack is assembled on high temperature tiles in one of the environmental test chambers.

The BMS and interface box are powered by an auxiliary 12 volt power supply. Figures 4 through 8 show the SAFT Li-Ion test setup.

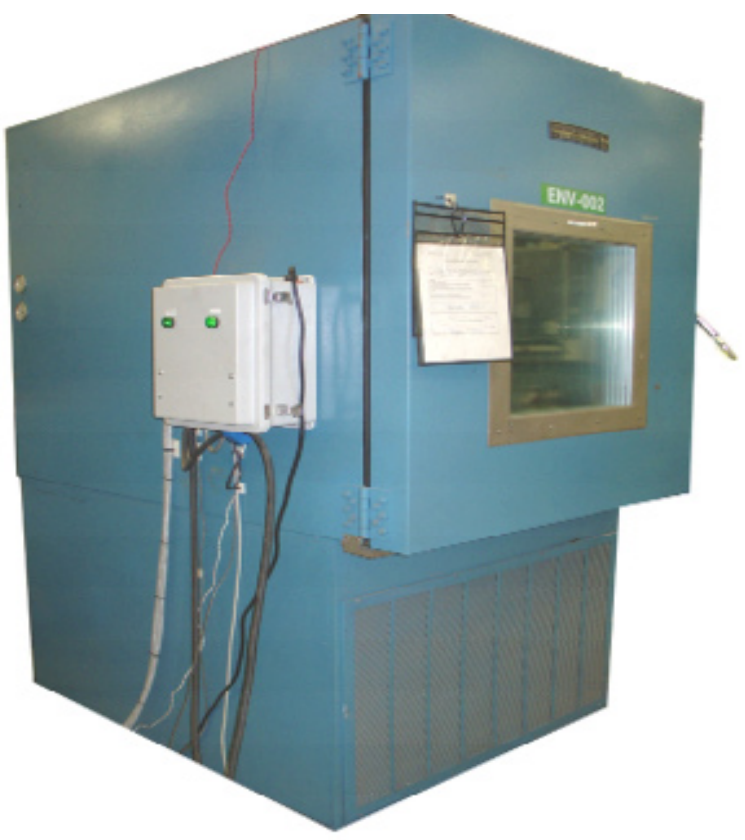

Figure 4: SAFT battery test chamber 


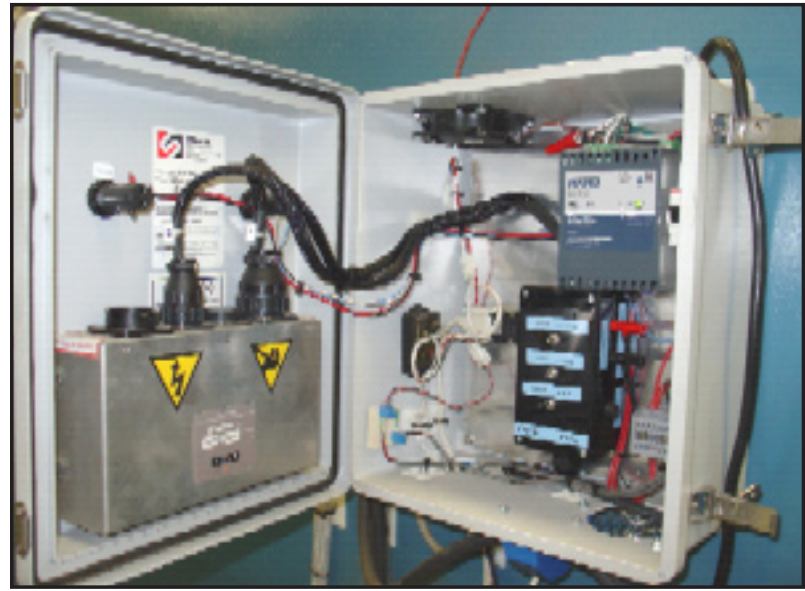

Figure 5: SAFT battery BMS and auxiliary power supply

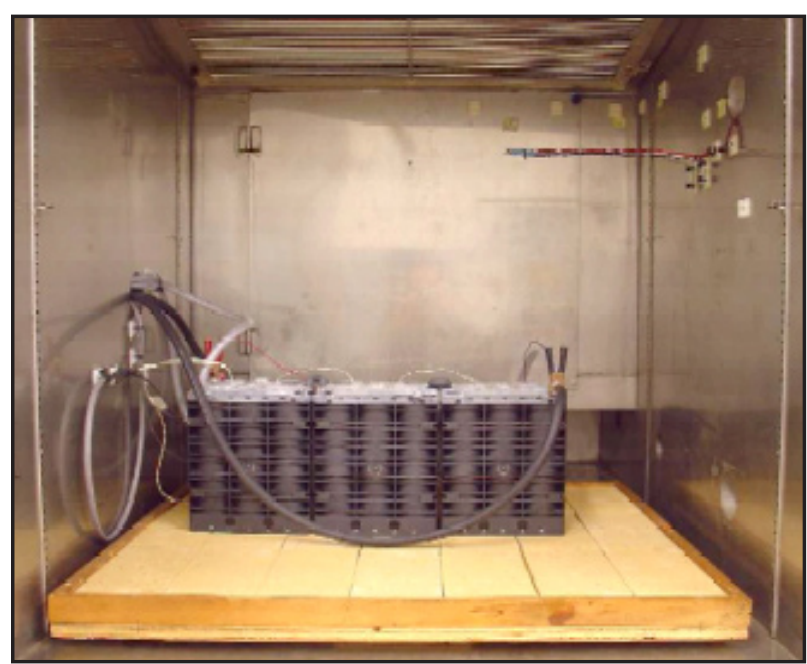

Figure 7: SAFT battery inside test chamber

3.2.1.2 Test Parameters

The test is conducted at an ambient temperature of $25^{\circ} \mathrm{C}$. The coolant fluid is maintained at a similar temperature. The battery modules are charged at a maximum current rate of $\mathrm{C} / 2$. A 30-minute rest period is allowed after the battery recharge.

If the cell voltage difference within the battery pack exceeds a specific threshold, the cell voltages have to be balanced. This action takes place automatically when the battery is not in use. The life cycle test will be paused to allow for module voltage balancing as required.

\section{TEST RESULTS}

\subsection{Test Result Presentation Template}

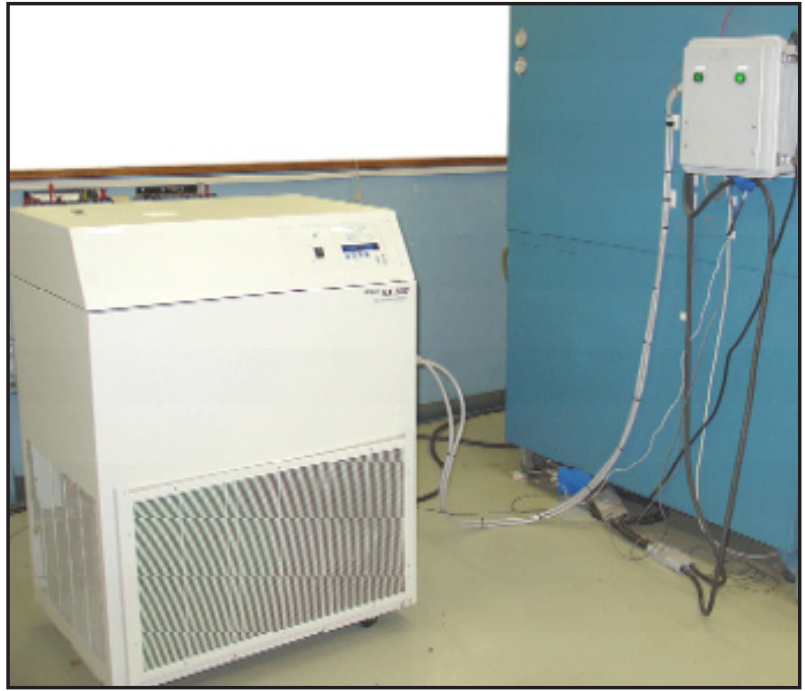

Figure 6: SAFT battery recirculation chiller

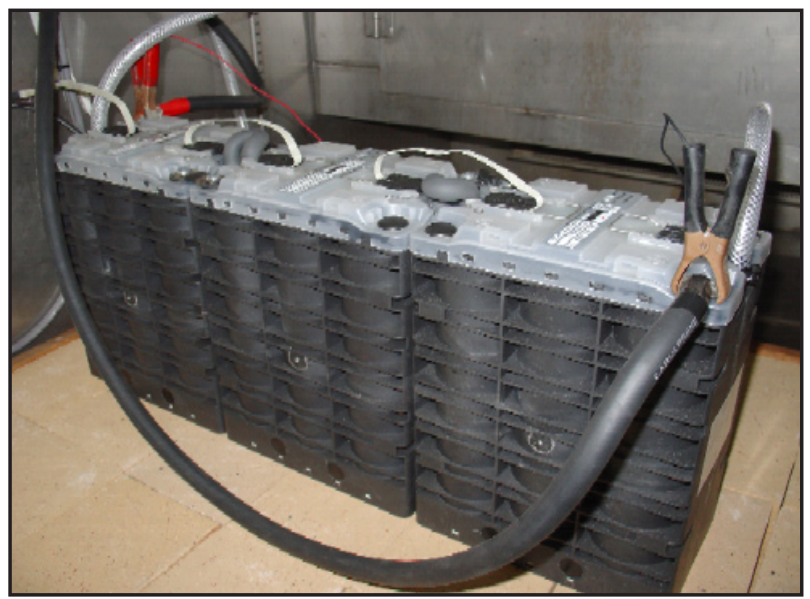

Figure 8: SAFT battery modules installed

To facilitate the data logging, the test cycles are logged in groups instead of being logged individually, as each battery test generates four test cycles of data per day. The number of test cycles per group can be adjusted manually or automatically.

Only the accuracy of the data collected from EVTCcalibrated test equipment is traceable to NIST. All other data are presented as provided by the battery manufacturer's BMS, with unspecified uncertainty.

\subsection{Battery Test Protocol Modification}

On April 7, 2006, the end of the charge depletion mode criteria was modified. Initially, the test profile was continuously applied to the battery until the battery voltage dropped below a pre-defined threshold for 10 consecutive seconds. This threshold had been 
determined at the beginning of the life cycle test to ensure that the battery SOC at the end of the charge depletion mode was approximately 25\%. However, after a year of testing, the capacity removed from the batteries during the charge depletion mode has drifted away from the $25 \%$ SOC target.

In order to be consistent with our original goal (depletion of the battery down to $25 \%$ SOC), the charge depletion mode termination criteria were changed. The mode is now ended when $75 \%$ of the battery manufacturer rated capacity has been removed, provided that none of battery manufacturer safety parameters are exceeded. This change occurred after 933 cycles have been applied to the SAFT battery pack.

\subsection{SAFT Li-Ion Battery Test Results}

\subsubsection{TEST EQUIPMENT ISSUE}

On cycle 718 , the cycling equipment channel used to test the SAFT battery pack failed. The entire cycler was replaced by a similar model recently calibrated. The following cycles showed inconsistent battery behavior with the previous 718 cycles. The test cycling equipment was replaced again with a new unit, the battery BMS and monitoring data acquisition cards were replaced too. However, the collected data were still inconsistent with previously gathered data. After complete investigation, it was found that the original battery cycler had drifted out of calibration. Since the unit failed, no calibration verification could be performed to assess the calibration error. The current, capacity and energy measurement were affected.

Based on the last set of data collected prior to the unit replacement and the first set of data collected with the calibrated unit, the measurement error was estimated and all previous affected data have been compensated.

\subsubsection{OVERVIEW}

Life cycle testing on the 3-module SAFT battery pack started on March 24, 2005. As of May 2007, 2103 test cycles have been applied to the battery. One initial RPT, eight scheduled RPTs and one manual RPT were performed. The data collected through cycle 719 show consistent battery performance throughout the three test modes. On cycle 720 the new calibrated test equipment was installed. Although previously collected data were compensated, the applied test profile is slightly different due to the previous current measurement error. Minor changes in the battery data graphs can be seen throughout the three test modes. In addition, the charge depletion termination criteria were modified on cycle 933 as described in paragraph
4.4

\subsubsection{TEST MODE RESULTS}

Figures 9 through 11 show the battery performance during the different test modes.

\subsubsection{REFERENCE PERFORMANCE TESTS}

Results from the RPTs performed are shown below.

Figure 12 shows the battery capacity as a function of the test cycle number.

The $\mathrm{C} / 1$ and $\mathrm{C} / 3$ battery capacity decreased respectively by $5 \%$ and $8 \%$.

Figure 13 shows the module peak power at specific DOD as a function of the test cycle number.

Approximately $7 \%$ at $80 \%$ DOD peak power degradation has been noticed thus far.

Figure 14 shows the battery pulse power capability on discharge and during a regenerative event at a specific DOD as a function of the test cycle number.

The battery power acceptance is limited by the BMS until the DOD reaches approximately $40 \%$.

Pulse power capability on discharge decreased by $6 \%$ at $80 \%$ DOD.

\subsection{Preliminary Conclusion}

The SAFT Li-Ion battery pack successfully performed through more than 2103 test cycles. The calibration errors encountered during the first 719 cycles have been addressed and should not affect the proper evaluation the battery. Nonetheless, limited battery performance degradation was noticed. Battery capacity decreased by $8 \%$ and battery power was reduced by $7 \%$. Collected data showed a linear degradation of the capacity and power performance of the battery.

The SAFT Li-Ion battery is showing encouraging results under PHEV applications.

It should be noted that one test cycle represents the theoretical daily operation of the Sprinter PHEV in the field (50-mile drive).

\section{TEST UPDATE}

This paper documents the work performed up to May 2007. Since, the battery reached 3700 cycles (equivalent 


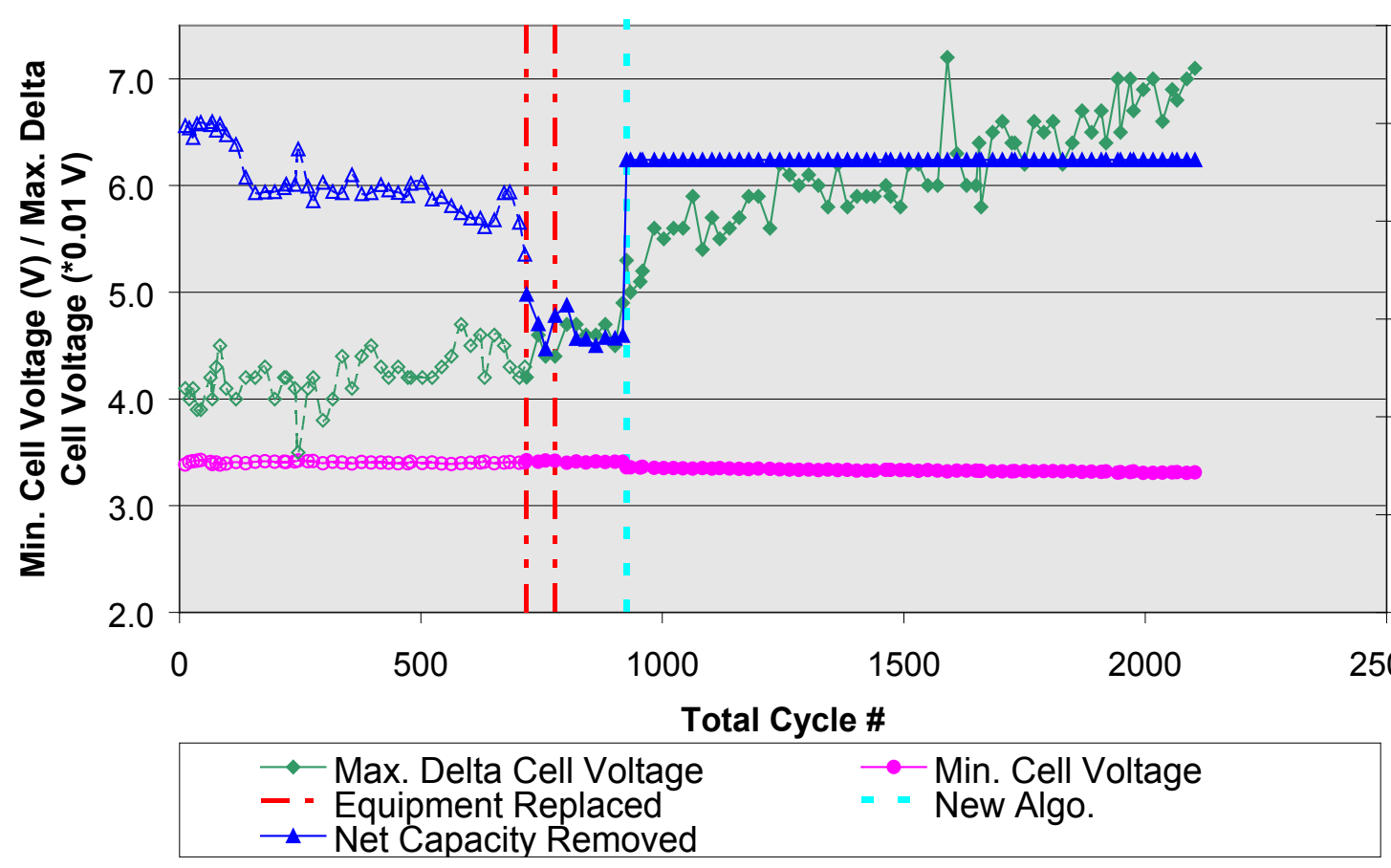

Figure 9: SAFT - charge depletion mode

Cell voltage data gathered using manufacturer BMS - Calibration certificate not made available

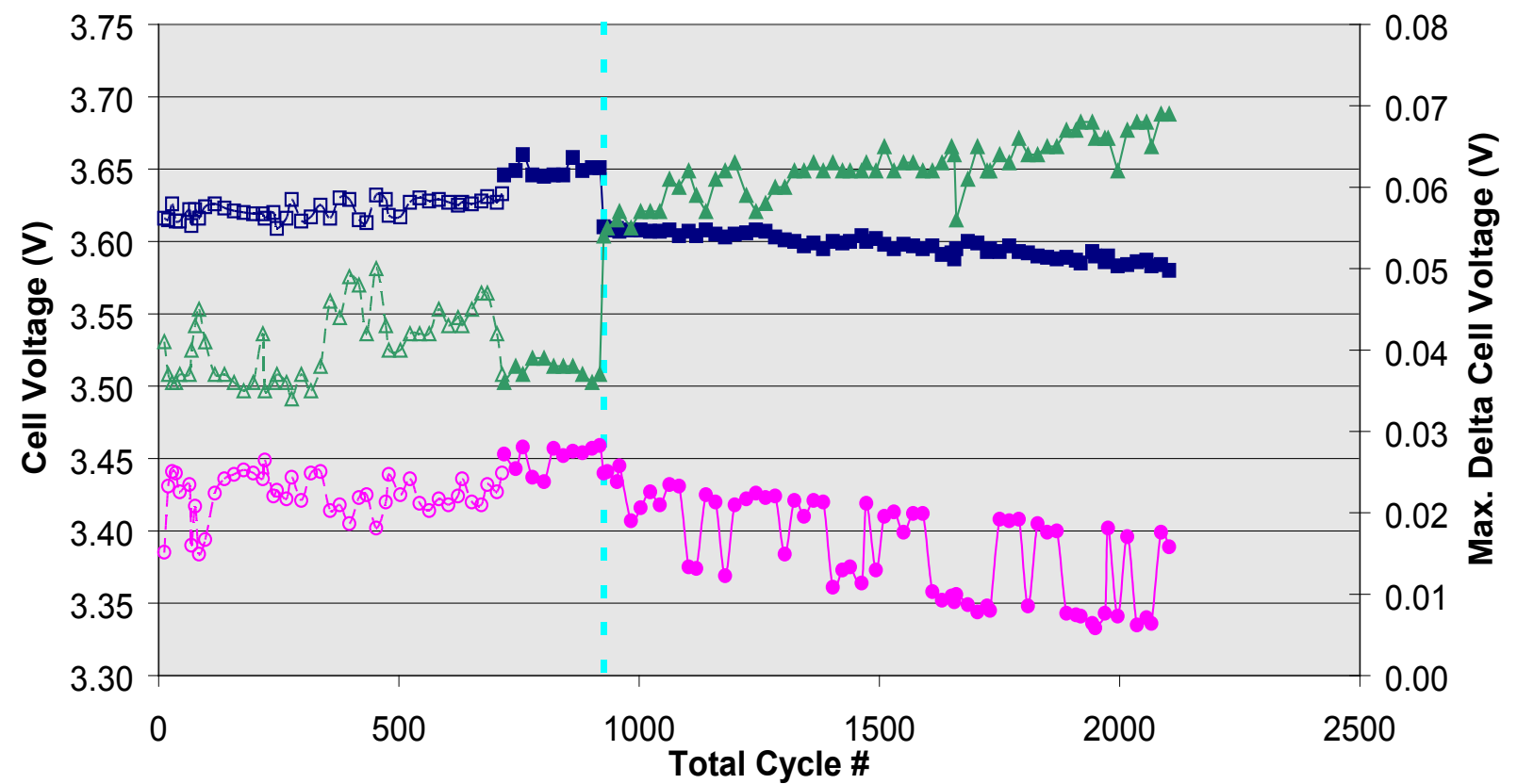

$\rightarrow$ Max. Cell Voltage New Algo.

Min. Cell Voltage

Max. Delta Cell Voltage

Figure 10: SAFT - charge sustained mode 


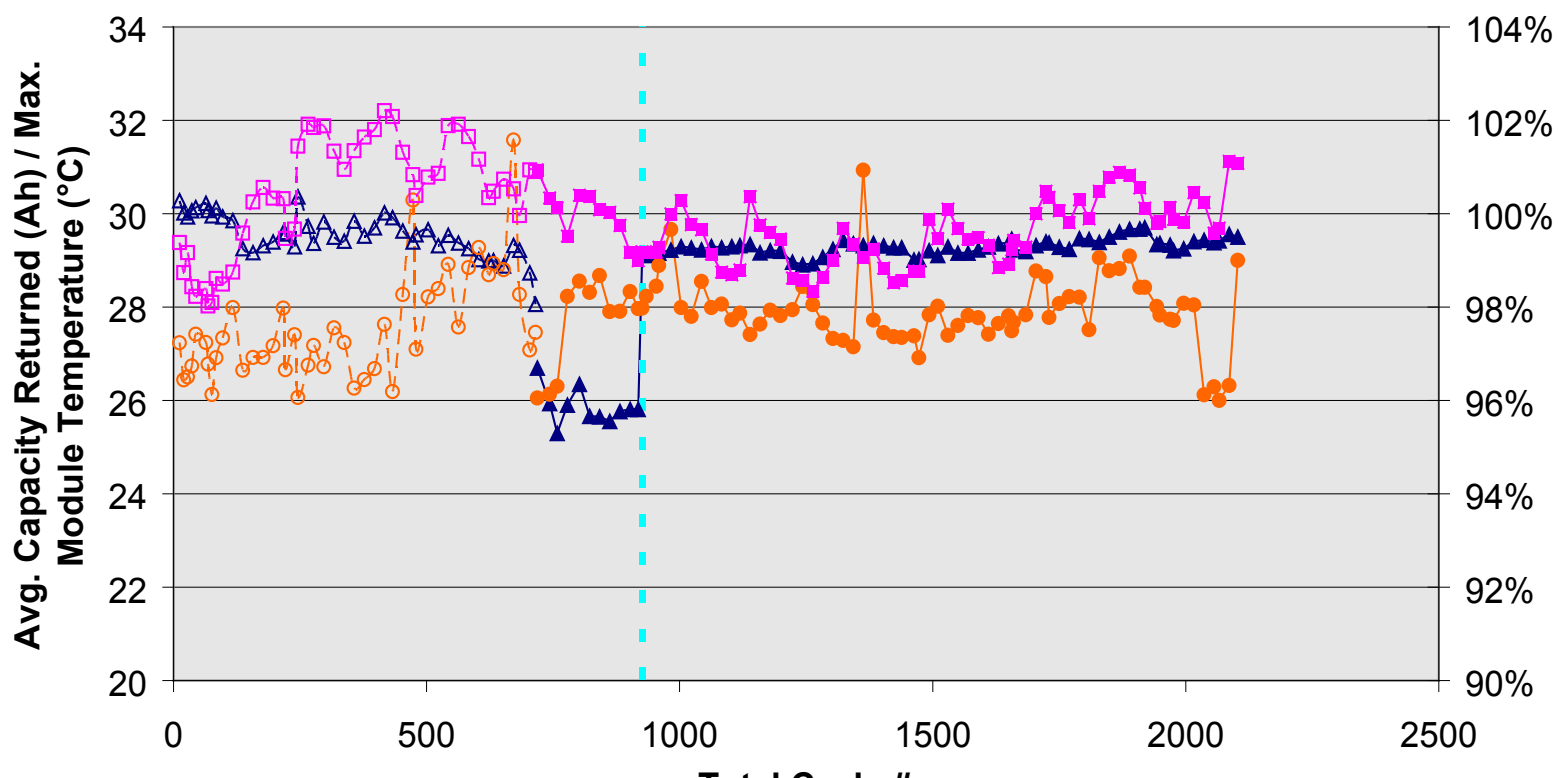

Total Cycle \#

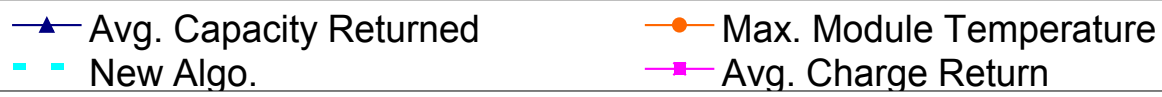

Figure 11: SAFT - recharge mode

Cell voltage data and temperature data gathered using manufacturer BMS - Calibration certificate not made available

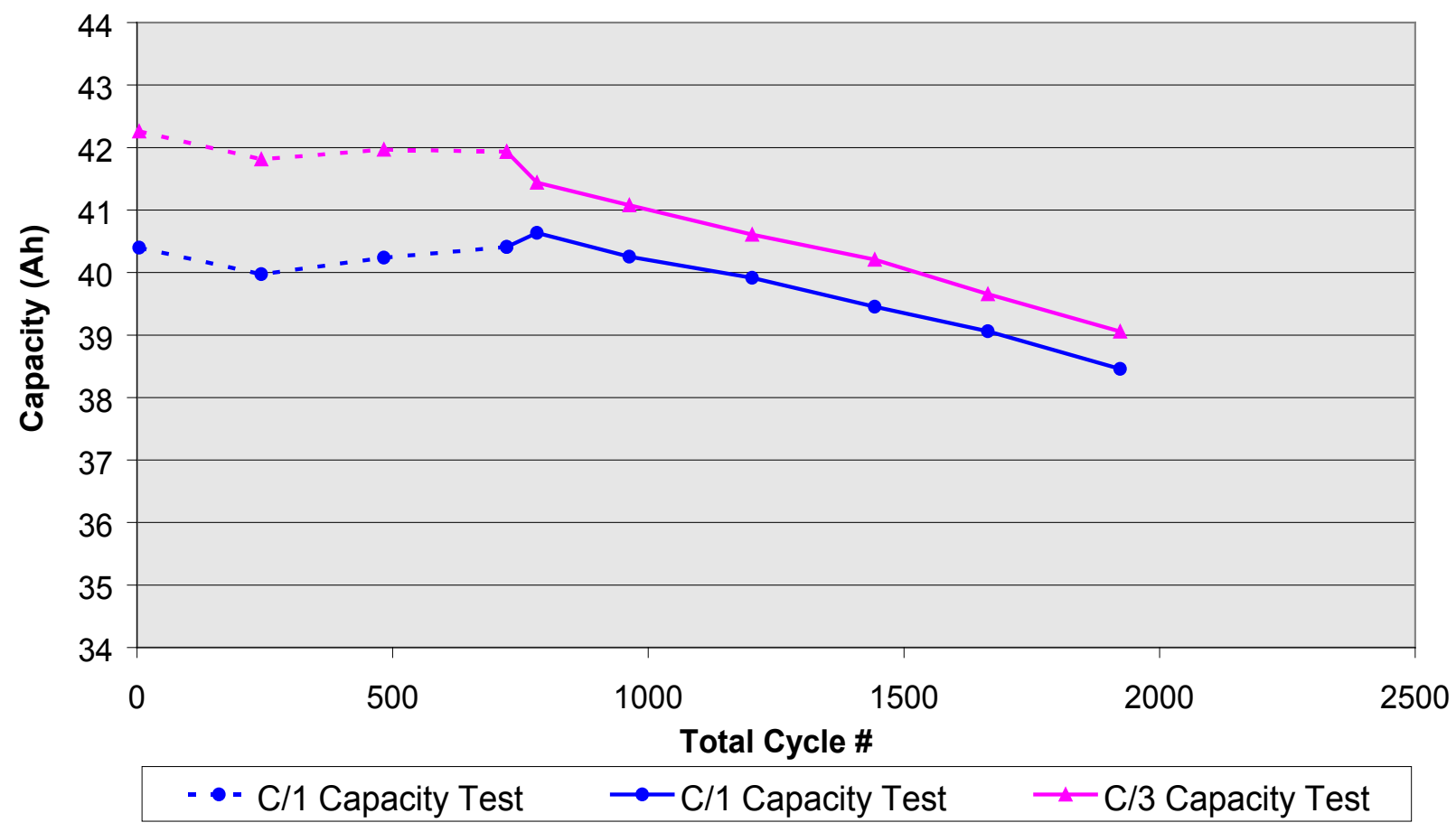

Figure 12: SAFT - battery capacity vs. cycle number 


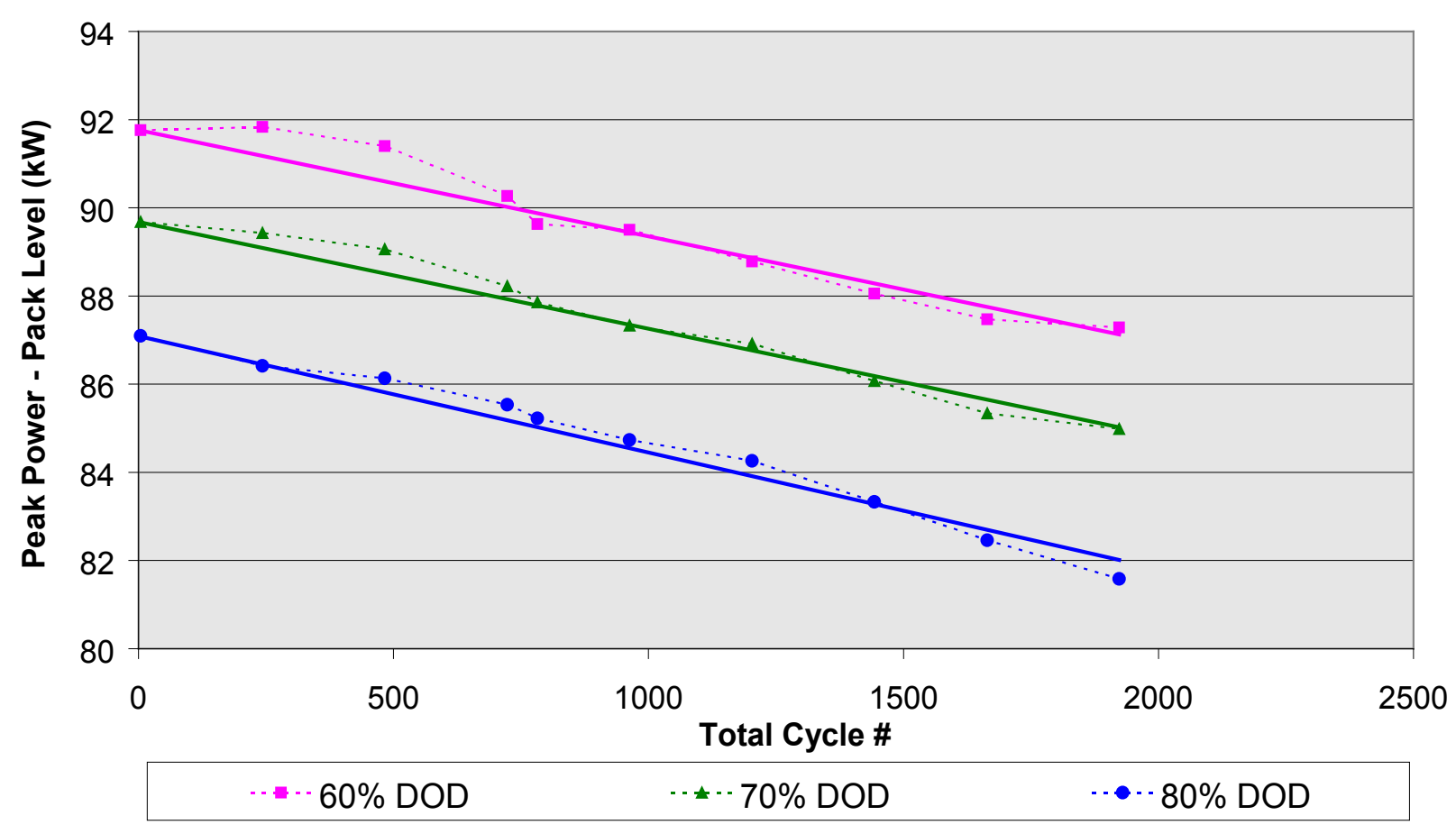

Figure 13: SAFT - peak power vs. cycle number



Figure 14: SAFT - HPPC test result 
to $185 \mathrm{k}$ miles of vehicle operation) and the test is still on-going.

\section{REFERENCES}

[1] USABC Electric Vehicle Battery Test Manual, Revision 2, January 1996.

[2] PNGV Battery Test Manual, Revision 3, February 2001.

\section{AUTHOR}

Loïc A. Gaillac is a project manager at SCE's Electric Vehicle Technical Center and evaluates alternativefuel vehicles, advanced energy storage systems (i.e. batteries, fuel cells, flywheels), and chargers in cooperation with manufacturers, government, and industry organizations. Before joining SCE, Mr. Gaillac was employed by PSA ("Peugeot Société Anonyme", a French automaker). Mr. Gaillac has a B.S. degree in Electrical Engineering and an M.S. degree in Power Electronics and Control Systems from a leading graduate school in France. 